\title{
Fatores de risco associados à perda dentária em idosos: uma revisão integrativa
}

\author{
Risk factors associated with tooth loss in the elderly: an integrative review \\ Factores de riesgo asociados con la pérdida de dientes en los ancianos: una revisión integrativa
}

Recebido: 24/08/2021 | Revisado: 29/08/2021 | Aceito: 01/09/2021 | Publicado: 05/09/2021

\author{
Ana Luzia Araújo Batista \\ ORCID: https://orcid.org/0000-0002-7780-060X \\ Universidade Estadual da Paraíba, Brasil \\ E-mail: analuziabatista@globo.com \\ Tatyanne Dias da Silva \\ ORCID: https://orcid.org/0000-0003-4349-981X \\ Universidade Estadual da Paraíba, Brasil \\ E-mail: tatydias.s1991@gmail.com \\ Taynná Larissa Santos Rodrigues \\ ORCID: https://orcid.org/0000-0002-4294-5470 \\ Universidade Estadual da Paraíba, Brasil \\ E-mail: tlsrodrigues1@gmail.com \\ Wanderson Ramon Barbosa Andrade \\ ORCID: https://orcid.org/0000-0002-7298-6994 \\ Universidade Estadual da Paraíba, Brasil \\ E-mail: wanderson1860@ hotmail.com \\ Wanessa Wanderleia Medeiros Silva \\ ORCID: https://orcid.org/0000-0002-2469-9413 \\ Universidade Estadual da Paraíba, Brasil \\ E-mail: wanessawmsilva@gmail.com \\ Ygor Alexandre Beserra de Sousa \\ ORCID: https://orcid.org/0000-0002-0690-521X \\ Universidade Estadual da Paraíba, Brasil \\ E-mail: ygooralexandre@hotmail.com \\ Maria Helena Chaves de Vasconcelos Catão \\ ORCID: https://orcid.org/0000-0001-7681-3225 \\ Universidade Estadual da Paraíba, Brasil \\ E-mail:mhcvcatao@gmail.com
}

\begin{abstract}
Resumo
O envelhecimento é um fenômeno que tem acontecido no mundo todo, resultando no aumento do número de idosos, que são consideradas pessoas maiores de 60 anos. Com esse fato, tem tido um aumento das condições crônicas, acompanhada no âmbito da saúde oral de uma considerável perda dentária. Portanto, o objetivo desse estudo foi realizar uma revisão integrativa da literatura a fim de analisar os fatores locais e sistêmicos, fisiológicos e patológicos que influenciam a perda dentária. Desse modo, foi realizada uma busca direcionada aos sites PUBMED, LILACS e SCIELO. Estabelecidos os critérios de inclusão e exclusão, ao final, dois artigos foram selecionados para discussão dos recursos metodológicos e achados mais relevantes. Pôde-se observar nessa busca que a maioria dos estudos são revisão de literatura nas mais diversas temáticas e que algumas pesquisas encontradas enfatizam algum fator específico. Portanto, é sabido que a perda dentária ainda é uma realidade nos idosos da atualidade e que esse público necessita de cuidados preventivos com a finalidade de manter a saúde oral, sistêmica e garantir uma qualidade de vida adequada.
\end{abstract}

Palavras-chave: Idoso; Perda de dente; Envelhecimento.

\begin{abstract}
Aging is a phenomenon that has happened all over the world, resulting in an increase in the number of elderly people, who are considered to be people over 60 years of age. With this fact, there has been an increase in chronic conditions, accompanied in the scope of oral health by a considerable tooth loss. Therefore, the aim of this study was to carry out an integrative literature review in order to analyze the local and systemic, physiological and pathological factors that influence tooth loss. Thus, a search was conducted on the PUBMED, LILACS and SCIELO websites. Once the inclusion and exclusion criteria were established, at the end, two articles were selected for discussion of the most relevant methodological resources and findings. It could be observed in this search that most studies are literature reviews on the most diverse themes and that some research found emphasize some specific factor. Therefore, it is known that tooth loss is still a reality in the elderly today and that these public needs preventive care in order to maintain oral and systemic health and ensure an adequate quality of life.
\end{abstract}

Keywords: Aged; Tooth los; Aging. 


\section{Resumen}

El envejecimiento es un fenómeno que se ha producido en todo el mundo, resultando en un aumento del número de personas mayores, que se consideran personas mayores de 60 años. Con este hecho, se ha producido un aumento de las enfermedades crónicas, acompañado en el ámbito de la salud bucal de una considerable pérdida de piezas dentarias. Por tanto, el objetivo de este estudio fue realizar una revisión integradora de la literatura con el fin de analizar los factores locales y sistémicos, fisiológicos y patológicos que influyen en la pérdida dentaria. Así, se realizó una búsqueda dirigida a los sitios web PUBMED, LILACS y SCIELO. Una vez establecidos los criterios de inclusión y exclusión, al final, se seleccionaron dos artículos para la discusión de los recursos y hallazgos metodológicos más relevantes. Se pudo observar en esta búsqueda que la mayoría de los estudios son revisiones de literatura sobre los más diversos temas y que algunas investigaciones encontradas enfatizan algún factor específico. Por tanto, se sabe que la pérdida de dientes sigue siendo una realidad en los ancianos en la actualidad y que este público necesita cuidados preventivos para mantener la salud bucal y sistémica y asegurar una adecuada calidad de vida.

Palabras clave: Anciano; Pérdida de diente; Envejecimiento.

\section{Introdução}

O processo de envelhecimento populacional tem se tornado crescente em todo o mundo. No Brasil, a população está envelhecendo rapidamente como consequência da redução na taxa de mortalidade e de fecundidade e do aumento da expectativa de vida. A estimativa é de que, até o ano de 2025, o Brasil seja o sexto país com o maior número de idosos, com mais de trinta milhões, o que representará um percentual aproximado de $15 \%$ da população total da nação (Silva et al., 2016).

Nos países desenvolvidos, este processo vem ocorrendo associado às melhorias nas condições gerais de vida. Contudo, nos países em desenvolvimento, tem ocorrido de forma rápida e sem tempo para uma reorganização social adequada, no sentido de atender às novas demandas emergentes, gerando implicações sociais em diferentes setores, sobretudo, em relação aos cuidados com a saúde (Silva et al., 2016). Dessa forma, o envelhecimento populacional constitui um novo desafio mundial para as políticas pública, especialmente nos países em desenvolvimento, como é o caso do Brasil (Melo et al., 2017).

O envelhecimento é um processo fisiológico e natural com modificações morfológicas, funcionais, bioquímicas e psicológicas que podem ocasionar comprometimento da autonomia e adaptação dos indivíduos diante do meio externo, tornando-os mais vulnerável a diversas patologias (Macena, Hermano \& Costa, 2018). As alterações patológicas também se modificam com o passar da idade, saindo de um quadro predominante de doenças infecciosas e parasitárias, que atingem em sua maioria os mais jovens, passando para um cenário de doenças crônicas e degenerativas em idosos (Oliveira, 2019).

Com relação à saúde bucal, a atenção odontológica, suas estratégias e cuidados suscitam debates específicos em torno de uma proposta de aperfeiçoamento do modelo assistencial ao idoso, na medida em que, ao envelhecer, é natural que o indivíduo esteja mais propenso a apresentar sintomas de doenças bucais que, na ausência de tratamento, poderão trazer consequências gravíssimas para sua saúde sistêmica (Silva et al., 2016).

Os cuidados com a saúde oral requerem atenção especial para a sua manutenção tendo em vista que na velhice os reflexos e coordenação do idoso são bem mais reduzidos, acrescentando-se a resistência do idoso em aceitar o tratamento indicado, fazendo-se necessário, nesse caso, que o profissional possua habilidades especiais e poder de persuasão para convencer da necessidade real (Silva et al., 2016).

Nesse cenário, dando ênfase ao sistema estomatognático, destacam-se as doenças intraorais, como a cárie dentária e a doença periodontal, contribuindo para o declínio da saúde bucal que, quando associada aos vários fatores de risco, dentre eles os modificadores de ordem sistêmica, contribuem para a perda dentária (Kim et al., 2019; Konishi, Verdonschot \& Kakimoto, 2020).

A elevada perda dentária atualmente encontrada nos idosos brasileiros é resultado do histórico de um modelo assistencialista com ênfase nas práticas curativas e mutiladoras, limitadas tão somente ao reparo de sequelas. No entanto, esse fenômeno do envelhecimento populacional exige mudanças sociais profundas como a necessidade de estabelecer padrões 
aceitáveis de saúde bucal, de modo a contribuir para melhorias no estado geral de saúde e bem-estar do paciente (Figueiredo, Carvalho \& Carvalho, 2019).

A perda dentária é um dos fatores que levam à fragilidade oral e a perda de funções, como estética, fonética, mastigatória, nutricional, social o que reflete na qualidade de vida dos idosos (Kim et al., 2019; Konishi, Verdonschot \& Kakimoto, 2020). Portanto, mediante o fenômeno de envelhecimento mundial e diante do contexto de saúde oral, o presente artigo tem como objetivo a realização de uma revisão integrativa para analisar os fatores relacionados à perda dentária em idosos, que pode vir a comprometer além da condição de saúde local, a condição sistêmica e consequentemente a qualidade de vida do público-alvo em questão.

\section{Metodologia}

Trata-se de uma revisão integrativa de literatura que, de acordo com Whittemore \& Knafl (2005) é a mais ampla abordagem metodológica referente às revisões para uma compreensão completa do fenômeno, no estudo em questão, a temática da perda dentária em idosos.

Para o levantamento dos artigos na literatura, realizou-se uma busca, no período entre março e abril de 2021, nas seguintes bases de dados: SCIELO, PUBMED e LILACS, utilizando as seguintes palavras-chaves "tooth loss" AND "elderly patients". Os critérios de seleção definidos para a seleção do artigo foram: artigos sem restrição de idioma, com apresentação na íntegra, que retratassem a temática referente à revisão integrativa, do tipo ensaio clínico e publicados e indexados nos referidos bancos de dados nos últimos 5 anos.

\section{Revisão de Literatura}

\section{Idoso e o processo de envelhecimento}

O envelhecimento, de acordo com a Organização Pan-Americana de Saúde (OPAS), é definido como um "processo sequencial, individual, acumulativo, irreversível, universal, não patológico de deterioração de um organismo maduro, próprio a todos os membros de uma espécie (Chagas \& Rocha, 2012), com ocorrência de mudanças psicológicas, biológicas e sociais, variando de acordo com questões genéticas, estilo de vida, meio ambiente e alimentação (Ferreira, 2010). Idoso, por sua vez, conforme definição do Estatuto do idoso, é todo indivíduo com faixa etária igual ou superior a 60 anos, de ambos os sexos, sem distinção de cor, raça e ideologia.

No Brasil, o aumento da expectativa de vida é crescente nas últimas décadas, migrando de um perfil demográfico característico de uma população jovem para um cenário no qual ocorre um crescimento em faixas etárias mais avançadas (Rodrigues, 2016). Em 1950, a população idosa representava 4,9\% da população do país, já em 2000, esse número passou a representar 7,9\%, em 2010 saltou para 10,2\% e em 2040 essa porcentagem pode chegar a 23,9\%. Desse modo, esse fenômeno causará mudanças no perfil epidemiológico do país, com uma maior incidência de doenças crônicas, desencadeando assim, altos custos para o sistema de saúde (Firmo, 2020).

A medida que a população mundial está constantemente envelhecendo, sua qualidade de vida relacionada à saúde revela uma preocupação com a Saúde Pública (Burci et al., 2020). Nessa conjuntura, faz-se necessário criar ações voltadas à saúde do idoso, que assegurem a melhoria da qualidade de vida no processo de envelhecimento. Para isso, no Brasil, existem a Política Nacional do Idoso (Lei 8.842) e o Estatuto do Idoso (Lei 10.741) que garantem direitos sociais, de modo a assegurar uma participação efetiva na sociedade (Pereira, Lacerda \&Natal, 2017).

Em se tratando da Odontologia, a manutenção de uma boa saúde bucal é um componente importante para se alcançar um envelhecimento saudável (Dahl, Calogiuri \& Jonsson, 2018). No Brasil, o estado atual de saúde bucal dos idosos é marcado 
por uma perda dentária elevada, consequente de um modelo assistencial curativo e reparador que se instaurou ao longo dos anos (Figueiredo, Carvalho \& Carvalho, 2019).

A relação entre saúde bucal e saúde geral é particularmente visível entre as pessoas idosas. A pobre condição de saúde oral é refletida particularmente em altos níveis de perda dentária, que por sua vez pode influenciar a saúde geral em termos de perda de peso, problemas alimentares e desvantagem social. Além do mais, reduz o desempenho mastigatório e limita a escolha alimentar, com efeitos emocionais consideráveis, tanto psicologicamente quanto socialmente (Burci et al., 2020).

\section{Alterações fisiológicas do idoso que contribuem para a perda dentária}

O envelhecimento fisiológico compreende uma série de alterações nas funções orgânicas devido exclusivamente aos efeitos da idade avançada sobre o organismo, com redução na capacidade de manter o equilíbrio homeostático e declínio gradual das funções fisiológicas (Chagas \& Rocha, 2012).

As disfunções encontradas nos idosos podem ser interpretadas como fruto de excessiva demanda imposta a um sistema fisiologicamente incapaz de supri-las e/ou pela existência de processos patológicos que, embora geralmente camuflados, nesta faixa etária, merecem toda atenção diagnóstica e terapêutica. Os indivíduos idosos apresentam alterações nos diversos sistemas, dentre eles, o mastigatório, do qual a cavidade oral faz parte (Silva, 2011).

Em geral, todos os tecidos da cavidade oral sofrem atrofia e perda de elasticidade, desde a mucosa até estruturas ósseas, passando pelos tecidos de sustentação e estruturas musculares. Algumas alterações são observadas em consequência das manifestações de doenças sistêmicas, deficiências nutricionais, efeitos colaterais pelo uso de fármacos, repercutindo no funcionamento dos tecidos periodontais, na dentição, nas glândulas salivares e mucosas orais (Silva, 2011).

Nos idosos, ocorre uma perda linear na quantidade de células acinosas, com uma redução de aproximadamente 20 a $30 \%$ na capacidade funcional das glândulas salivares, responsáveis pela produção de saliva, dando lugar a tecido conjuntivo ou gordura e resultando na diminuição no volume da secreção salivar (Freitas Jr et al., 2008; Silva, 2011; Lopes et al., 2021).

$\mathrm{O}$ avanço da idade também promove uma alteração gradual no tecido ósseo, com diminuição do tecido mineralizado, onde a reabsorção aumenta e a neoformação óssea diminui, resultando em um aumento da porosidade óssea (Freitas Jr et al., 2008; Lopes et al., 2021). A massa óssea é maior nos homens do que nas mulheres, mas com a perda progressiva, os homens têm uma redução de 3,3\% ao ano e ao mulheres de $1 \%$, sendo que no período pós-menopausa essa perda aumenta em até dez vezes (Chagas \& Rocha, 2012). Por meio de mecanismos semelhantes aos que ocorrem nas demais partes do organismo, as estruturas do sistema estomatognático são igualmente afetadas pelo processo de envelhecimento (Freitas Jr et al., 2008).

O periodonto dos idosos fica comprometido, sendo afetado tanto o ligamento periodontal como o processo alveolar. As fibras periodontais alteram sua disposição prejudicando as propriedades mecânicas, além do mais ocorre um aumento na produção de prostaglandinas, favorecendo a inflamação e uma maior liberação do fator ativador dos osteoclastos. Acrescentese a isso, a diminuição da reatividade do tecido conjuntivo associada a uma baixa capacidade defensiva diante da agressão bacteriana, pela depressão imunitária e dos fagócitos, além de uma diminuição da queratinização na gengiva e aumento na largura da gengiva inserida (Freitas Jr et al., 2008).

Essa perda da morfologia gengival, torna a mucosa oral atrofiada e friável, em decorrência da diminuição da espessura média do epitélio, de alterações na camada basal da mucosa, da redução do grau de queratinização das camadas superficiais e do suprimento sanguíneo, prejudicando o processo de renovação e regeneração tecidual, estando mais sujeita a injúrias e promovendo uma lenta cicatrização (Freitas Jr et al., 2008; Lopes et al., 2021).

O sistema nervoso é o sistema fisiológico mais comprometido com o processo do envelhecimento, sendo responsável por diferentes tipos de sensação, movimentos, funções psíquicas, entre outros. As alterações mais importantes, características 
do envelhecimento, ocorrem no cérebro que diminui de peso e tamanho, estando a redução de massa associada à perda neuronal e certo grau de atrofia cortical (Chagas \& Rocha, 2012).

A idade avançada pode alterar a habilidade para realizar a higiene oral, seja por deficiência física ou por falta de motivação (Silva, 2011). A demência, síndrome multietiológica responsável por distúrbios de memória, mudanças de personalidade e prejuízos ao raciocínio pode levar ao impedimento da realização de tarefas cotidianas, com consequente perda da autonomia dos indivíduos (Ranjan et al., 2019).

Alguns idosos apresentam maior risco de desenvolver transtornos mentais e os sintomas de sofrimento psicológico são comuns e graves. Como consequência, causam perturbação significativa na vida diária, levando a uma redução da qualidade de vida, com impacto negativo sobre os comportamentos relacionados à saúde, incluindo seus padrões alimentares bem como seus comportamentos de higiene dental, que por sua vez, pode levar a problemas de saúde bucal (Dahl, Calogiuri \& Jonsson, 2018).

A redução da capacidade gustativa é verificada a partir dos cinquenta anos e atinge $80 \%$ dos pacientes idosos e essas alterações estão associadas a redução do número de botões gustativos, fato que afeta diretamente a nutrição, uma vez que o alimento passa a ser menos apetitoso e desejado pelo idoso (Lopes et al., 2021).

No processo de envelhecimento o corpo humano sofre alterações fisiológicas consideráveis, sendo necessário que o Cirurgião-Dentista tenha ciência dessas mudanças uma vez que a maioria delas acomete a saúde bucal. É necessário conhecer as alterações fisiológicas e patológicas que acometem o organismo do paciente idoso, bem como os aspectos psicossociais que influenciam na qualidade de vida (Lopes et al., 2011).

\section{Alterações patológicas do idoso associadas à perda dentária}

Doenças relacionadas ao envelhecimento nos países ocidentais estão se tornando cada vez mais prevalentes com um impacto socioeconômico significativo devido ao aumento da média de vida (Dioguardi et al., 2019). A maioria dos problemas odontológicos observados na população da terceira idade está relacionada com complicações patológicas adquiridas no decorrer da vida, associadas ou como resultado de alterações fisiológicas com o passar do tempo, da ineficiente higiene bucal, iatrogenia de profissionais, falta de orientação e de acesso aos serviços de saúde, seja por negligência pessoal ou condição socioeconômica (Silva, 2011; Lopes et al., 2021).

Dentre as alterações percebidas na cavidade oral do paciente idoso algumas são decorrentes de manifestações de doenças sistêmicas com repercussão na cavidade oral. Dentre as condições patológicas, as que apresentam maior prevalência e acometimento na saúde bucal do idoso, a cárie dentária e doença periodontal continuam em destaque, culminando com a perda dentária (edentulismo), acompanhadas pelas lesões de tecido mole (Catão \& Xavier, 2011; Dahl, Calogiuri \& Jonsson, 2018; Lopes et al., 2021).

Desse modo, vários fatores locais e doenças sistêmicas podem estar associados com a causa da perda dentária (Kim et al., 2019; Konishi, Verdonschot \& Kakimoto, 2020). A perda dentária é um dos fatores que levam à diminuição da função oral e consequentemente à sua fragilidade, assim como do corpo todo. Vale ressaltar ainda os fatores sociodemográficos como local que reside, renda e histórico educacional. Portanto, é fundamental que o idoso evite perdas dentárias a fim de prevenir o agravamento das funções orais (Konoshi, Verdonschot \& Kakimoto, 2020).

Uma das principais causas da perda dentária é a doença periodontal na qual a periodontite, sua forma mais agravante, afeta o aparelho de suporte dentário, levando à destruição dos tecidos moles e à reabsorção do osso alveolar (Dioguardi et al., 2019). A reabsorção do tecido ósseo alveolar é induzida por reações inflamatórias que ocorrem em resposta à infecção localizada devido à má higiene oral (Savić Pavičin et al. 2017; Taguchi et al., 2019; Tanaka et al. 2020).

A reabsorção óssea provocada pela periodontite pode ser agravada nos casos de osteoporose que é caracterizada pela diminuição da densidade óssea (Savić Pavičin et al. 2017; Taguchi et al., 2019; Takana et al., 2020). Em se tratando de 
mulheres na pós-menopausa, as complicações ósseas são ainda maiores, pois reduz os hormônios femininos que por sua vez controlam o metabolismo ósseo (Konoshi, Verdonschot \& Kakimoto, 2020).

Outro fator que afeta as estruturas ósseas, incluindo problemas que envolvem a articulação temporomandibular (ATM), é em decorrência do maior nível de estresse proporcionado pelo cotidiano deste século. A dor miofacial e o espasmo muscular são capazes de limitar o movimento da mandíbula, com queixa de bruxismo e apertamento dental, assim como presença de osteoartrite que pode induzir o aumento da degeneração da ATM, reduzindo a amplitude vertical de abertura bucal (Freitas Jr et al., 2008).

Um outro comprometimento da perda dentária pela periodontite diz respeito à diminuição na capacidade mastigatória do paciente, repercutindo diretamente nas funções do sistema estomatognático (Lopes et al., 2021) e podendo levar a uma dieta inadequada (Dioguardi et al., 2019). A função mastigatória é um fator importante para a nutrição e a perda de dentes, seja de seis pares de dentes ocluídos ou perda de oclusão de molares, mantendo no mínimo 20 dentes, pode induzir a seleção inadequada de alimentos, propiciar uma adaptação da textura dos alimentos ao estado dentário, acarretando uma diminuição do apetite assim como distúrbios de deglutição e, por fim, resultar em um quadro de desnutrição (Poisson et al., 2016; Konoshi, Verdonschot \& Kakimoto, 2020).

A deficiência na função mastigatória pode resultar na diminuição no fluxo salivar, estando relacionado ao hipercatabolismo e associada à disfagia crônica. Essa redução da saliva pode também ser resultado do uso de determinadas medicações (antidepressivos, tranquilizantes e anti-histamínicos), ventilação oral crônica ou presença de algumas patologias, como diabetes, síndrome de Sjogren e insuficiência renal (Poisson et al., 2016).

Esse quadro de xerostomia, ou seja, a diminuição no volume da secreção salivar, deixa os tecidos bucais mais susceptíveis a alterações patológicas, prejudicando a formação do bolo alimentar e a fonação, podendo ainda acarretar o aparecimento de cáries rampantes, candidíase, desconfortos na mastigação, disfagia, dificuldades para usar próteses e na percepção do gosto dos alimentos, estando associada também aos efeitos colaterais do tratamento do câncer e como consequência da desidratação (Freitas Jr et al., 2008; Silva, 2011; Lopes et al., 2021).

Outro fator agravante para a perda dentária em idosos são os transtornos psiquiátricos que podem vir acompanhado de outros problemas como condições socioeconômicas, doenças sistêmicas, desequilíbrio do estado mental, dificuldade de acesso ao tratamento odontológico associado à sobrecarga dos familiares e cuidadores, ao despreparo de grande parte dos profissionais, às deficiências na higiene oral, além de efeitos colaterais causados por medicamentos psicotrópicos (Figueiredo, Carvalho \& Carvalho, 2019).

Essa diminuição das funções intelectuais e consequentemente a redução da habilidade manual, dificulta a higiene oral e agrava as condições patológicas intraorais preexistentes (Dioguardi et al., 2019). Portanto, um desafio em se tornar idoso é manter boa saúde bucal, especialmente para idosos frágeis ou com deficiência cognitiva, por ser um componente importante da boa condição oral (Dahl, Calogiuri \& Jonsson, 2018).

\section{Resultados e Discussão}

Com a busca direcionada, foram selecionados 34 artigos, sendo 15 artigos pela PUBMED e 19 artigos pelo LILACS. Nenhum artigo foi encontrado no SCIELO. Por meio da conferência pelo título, foram excluídos 9 artigo do PUBMED e 10 artigos do LILACS, totalizando 19 e ficando 15 para leitura do resumo. Nessa etapa seguinte, 6 artigos estavam em duplicata, sendo 2 deles os selecionados para a análise metodológica e discussão dos achados mais relevantes (Quadro 1). 
Quadro 1. Informações detalhadas de cada estudo referente ao título do artigo, autores, ano, detalhes metodológicos e principais conclusões.

\begin{tabular}{|c|c|c|c|}
\hline Autor, ano e título & Objetivo & Metodologia & Principais conclusões \\
\hline $\begin{array}{c}\text { Ranjan et al., } 2019 \\
\text { Perda de dente e } \\
\text { demência: uma } \\
\text { conexão oro-neural. } \\
\text { Um estudo } \\
\text { transversal }\end{array}$ & $\begin{array}{l}\text { Estabelecer a correlação entre } \\
\text { perda dentária e diagnóstico de } \\
\text { comprometimento cognitivo / } \\
\text { demência em pacientes idosos }\end{array}$ & $\begin{array}{c}\text { Estudo transversal } \\
300 \text { pacientes } \\
\text { (entre } 50 \text { e } 80 \text { anos) } \\
\text { Teste do mini-exame do estado } \\
\text { mental (MEEM) - avaliação de } \\
\text { comprometimento } \\
\text { cognitivo/demência }\end{array}$ & $\begin{array}{c}\text { Há uma associação significativa entre } \\
\text { a perda dentária e o } \\
\text { comprometimento cognitivo/ } \\
\text { demência. } \\
\text { Indivíduos com diagnóstico de } \\
\text { demência grave e perda dentária } \\
\text { apresentam reflexos na saúde } \\
\text { periodontal, ou seja, a periodontite } \\
\text { parece estar associada ao } \\
\text { comprometimento cognitivo. }\end{array}$ \\
\hline $\begin{array}{c}\text { Konishi, } \\
\text { Verdonschot, } \\
\text { Kakimoto, 2020 } \\
\text { Uma investigação } \\
\text { dos fatores de perda } \\
\text { dentária em } \\
\text { pacientes idosos } \\
\text { usando } \\
\text { radiografias } \\
\text { panorâmicas }\end{array}$ & $\begin{array}{l}\text { Observar a condição dentária em } \\
\text { um grupo de idosos ao longo de } \\
\text { um período de } 10 \text { anos, a fim de } \\
\text { esclarecer fatores de risco } \\
\text { importantes. }\end{array}$ & $\begin{array}{l}\text { Comparação de achados em } \\
\text { radiografias panorâmicas em um } \\
\text { período de } 10 \text { anos. } \\
\text { Foram avaliados a condição de } \\
\text { saúde oral, considerando: número } \\
\text { de dentes, índice de Eichner, } \\
\text { oclusão, condição pulpar e } \\
\text { periapical, reabsorção óssea } \\
\text { alveolar e próteses. }\end{array}$ & $\begin{array}{l}\text { Os resultados demonstram que o } \\
\text { número de dentes diminuiu com o } \\
\text { avançar da idade. Os autores } \\
\text { concluíram que dentes que mostram } \\
\text { uma maior reabsorção, presença de } \\
\text { próteses e lesões, apresentam maior } \\
\text { probabilidade de perda no futuro } \\
\text { próximo de um paciente idoso. }\end{array}$ \\
\hline
\end{tabular}

Fonte: Autores.

Como amplamente divulgado na literatura, o processo de envelhecimento é acompanhado do agravamento das perdas dentárias, sendo as principais causas a cárie dentária e a doença periodontal. Além das causas locais, várias doenças sistêmicas também exercem sua influência, tais como, hipertensão, doenças cardíacas, diabetes mellitus, alterações hormonais e osteoporose pós-menopausa. Vale ressaltar ainda os fatores sociodemográficos como renda e histórico educacional (Konoshi, Verdonschot \& Kakimoto, 2020).

No estudo de Konishi, Verdonschot \&Kakimoto (2020) foi realizada uma investigação dos fatores de perda dentária em pacientes idosos usando radiografias panorâmicas ao longo de um período de 10 anos. Para tanto foram avaliados o número de dentes restantes e perdidos, o índice de Eichner, a presença ou ausência de oclusão molar, a condição da polpa dentária, a presença de coroas, reabsorção óssea alveolar e lesões periapicais. Os resultados demonstram que o número de dentes remanescentes diminuiu de uma média de 20,8 para 15,5 e a porcentagem de pacientes com 20 ou mais dentes diminuiu de 69,2 para 26,9\%. Os autores concluíram que dentes que mostram reabsorção óssea com envolvimento da crista óssea entre $1 / 3$ e $1 \frac{1}{2}$ da raiz ou maior, a presença de coroas e de lesões apicais apresentam maior probabilidade de perda no futuro próximo.

Com relação à reabsorção óssea, Freitas Jr et al. (2008) afirmar que, com o avançar da idade, os maxilares e a mandíbula sofrem atrofia por desuso, assim como aumenta a porosidade cortical da mandíbula e, dessa forma, o aumento da perda dentária pode estar associado à reabsorção óssea decorrente da idade que pode se agravar no rebordo residual dos pacientes edêntulos. Isso pode ser agravado devido ao acúmulo de biofilme e a presença de cálculo, característico da doença periodontal e pela existência de uma oclusão inadequada. Shen et al. (2020) ainda enfatizam que a perda da quantidade de osso maxilar pode estar envolvida com a reabsorção do osso alveolar induzida pela periodontite, do osso alveolar residual e da osteoporose maxilofacial que está relacionada com a osteoporose sistêmica. Esses autores concluem que a perda óssea pode causar reabsorção do osso alveolar e atrofia da mandíbula, comprometendo o prognóstico para o paciente idoso. 
A osteoporose tem associação com a perda dentária por ser um fator que exacerba a periodontite. Essa condição é mais provável de se desenvolver em mulheres na menopausa pela ocorrência da redução dos hormônios femininos que, por sua vez, controlam o metabolismo ósseo (Konoshi, Verdonschot \& Kakimoto, 2020). Além do mais, de acordo com Freitas Jr et. (2008), o hormônio da paratireóide e a vitamina D, que regulam a função dos ossos, rins e intestinos para manter a concentração normal de cálcio e fosfato do sódio, têm sua ação e eficácia significativamente modificadas com a idade.

Penoni et al. (2017) reforçam que mulheres em pós-menopausa que são portadoras de osteoporose podem apresentar uma maior perda de inserção periodontal clínica quando comparadas com mulheres que possuem densidade mineral óssea normal. Entretanto, para Pan et al. (2019), a perda dentária não pode ser estritamente atribuída a alterações hormonais na menopausa e a baixa dimensão da massa óssea, devendo-se considerar a conscientização da higiene bucal como uma questão fundamental. Merchant (2017), realizando um estudo com um grupo de homens idosos também constatou que a baixa densidade mineral óssea estava associada ao aumento da perda dentária ao longo do tempo e que a prevenção sistêmica à perda óssea pode reduzir a perda de dentes.

O estudo transversal realizado por Ranjan et al. (2021) teve como objetivo estabelecer a correlação entre perda dentária e diagnóstico de comprometimento cognitivo / demência em pacientes idosos. Para sua execução, foram avaliados 300 pacientes, com idade entre 50 e 80 anos. Para a avaliação do comprometimento cognitivo / demência, foi realizado o MiniExame do Estado Mental (MEEM). Como resultados, os autores encontraram que o número de dentes estava diretamente associado ao MEEM, ou seja, quanto menor número de dentes, menor a pontuação no referido exame, independentemente de gênero. A conclusão desse estudo é que há uma associação significativa entre a perda dentária, com ênfase na periodontite e o comprometimento cognitivo.

Foi estimado que a periodontite é responsável por cerca de 50\% das exodontias realizadas em pacientes acima de 40 anos de idade. Além do mais, o quadro sistêmico do paciente pode favorecer a persistência do progresso da condição oral e por esse motivo foi encontrada uma associação muito clara entre perda dentária com histórico de doenças periodontais e doenças inflamatórias sistêmicas (Ranjan et al., 2021).

Com relação ao comprometimento local, Freitas Jr et al. (2008) afirmam que o grau de desorganização do periodonto aumenta com a idade além de que, dentre todas as estruturas bucais, o periodonto é a estrutura mais afetada durante o processo de envelhecimento. Além do mais, a diminuição da reatividade do tecido conjuntivo associada a uma baixa capacidade defensiva diante da agressão bacteriana, pela depressão imunitária e dos fagócitos, faz com que a periodontite tenha efeitos mais graves nos idosos, com aumento da prevalência e severidade. Esse fato proporciona mobilidade dental e a posterior ausência do dente da cavidade alveolar, fato que reduz a superfície oclusal anatômica e funcional, determinando insuficiência estomatognática pela reduzida área oclusal e seu desequilíbrio funcional.

No entanto, a presença de bactérias como componente etiológico primário da periodontite além de favorecer a inflamação local, pode levar ao comprometimento sistêmico. Isso acontece em função da invasão bacteriana ou dispersão no organismo de seus produtos (endotoxinas e citocinas) que pode, por exemplo, atingir o sistema nervoso central, levando à morte de células nervosas e possivelmente à perde de memória, caracterizando um quadro de demênica (Dioguardi et al., 2019; Ranjan et al., 2019).

Na revisão sistemática com metanálise de estudos caso-controle de Dioguardi et al. (2019) eles explicam como a perda dentária pode influenciar negativamente a função cognitiva de várias maneiras: uma das formas é por meio da inflamação induzida por doença periodontal que está associada ao aumento de agentes pró-inflamatórios que, uma vez no plasma, levam a processos inflamatórios que afetam o cérebro, contribuindo para o declínio cognitivo. Os autores ainda reforçam que a perda do dente resulta em disfunção mastigatória, o que leva a uma nutrição mais pobre e redução subsequente na perfusão sanguínea cerebral e, por conseguinte, uma diminuição nos níveis de acetilcolina e no número de células 
piramidais envolvendo o hipocampo. Desse modo, as manobras relacionadas à higiene oral e a prevenção da doença periodontal em idosos com deficit cognitivo pode estar reduzida, causando uma maior perda de elementos dentários.

Ranjan et al. (2019) apontam que os indivíduos com demência estão mais propensos a desenvolver quadros de saúde bucal precária devido as dificuldades para manutenção da higiene oral e a redução do número de visitas preventivas ao dentista. A associação entre saúde bucal e saúde geral é especialmente visível entre os idosos devido às fragilidades cognitivas e higiene bucal deficiente, uma vez que manter a boa saúde bucal é fator importante para um envelhecimento saudável (Dahl, Calogiuri \& Jonsson, 2018).

No estudo de Poisson et al. (2016), os pacientes com menos de sete pares de oclusão posterior tinham um risco maior de disfagia, uma vez que, durante a mastigação, a ausência de dentes posteriores retarda a formação de um bolo alimentar homogêneo e coeso tornado a deglutição mais difícil, o que também pode ser agravado pela diminuição na força da língua em pessoas mais velhas. Além do mais, a perda de oclusão posterior pode induzir atrofia da glândula salivar e diminuir o fluxo salivar. Silva et al. (2016) reforçam que essa falta de dentes leva as pessoas a modificarem seus hábitos alimentares e a optarem por alimentos mais macios, de fácil mastigação e, muitas vezes, de baixo valor nutritivo.

A idade avançada pode alterar a habilidade para realizar a higiene oral, seja por deficiência física ou por falta de motivação (Silva, 2011). Vale mencionar algumas condições sistêmicas que reduz a habilidade manual, como os casos de mal de Parkinson, artrite reumatóide, assim como transtornos cognitivos e/ou psiquiátrico que levam ao desinteresse e ao descuido com a própria vida. Com relação aos fatores sociodemográficos, o fato se agrava em se tratando da redução da renda após a aposentadoria, aos custos adicionais com a saúde e aos gastos com cuidadores.

Esses aspectos têm sido abordados no estudo de Ranjan et al. (2019), no tocante a perda dentária, revelando que essa condição pode estar associada a escolaridade, uma vez que os indivíduos desconhecem a importância de manter uma boa higiene oral, agravado também por problemas relacionados ao estresse, como resultado do estilo de vida moderno em que as pessoas se preocupam menos com a higiene oral.

Uma outra questão a ser abordada diz respeito ao produto da atividade secretora das glândulas salivares: a saliva fornece a proteção, lubrificando a mucosa e prevenindo a desmineralização e promovendo a mineralização dos dentes. As alterações nas glândulas salivares podem provocar xerostomia e diminuição na produção da amilase salivar, o que dificulta a digestão oral e a deglutição posterior do bolo alimentar (Chagas \& Rocha, 2012). Lopes et al. (2021) ainda acrescentam que essas modificações resultam em dieta pobre e má nutrição e, dessa forma, a mucosa seca faz com que o número de bactérias aumente levando a problemas gengivais assim como do número de cáries, além de gerar um incomodo no uso de próteses dentárias.

Na revisão sistemática de Dioguardi et al., (2019), para os idosos, a perda de elementos dentários, seja devido a causas relacionadas a processos cariosos ou relacionadas a problemas periodontais ou endodônticos, leva a uma diminuição da capacidade mastigatória e da qualidade de vida. Apesar destas condições que afetam os idosos com problemas tratáveis ou não, muitos não gozam do tratamento adequado. Em análise, supõe-se que os idosos com idade superior a 60 anos, não foram apresentados ao conceito de Odontologia preventiva em uma idade jovem e, portanto, não estão dispostos a isso na "terceira idade". Muitos ainda têm a opinião de que a perda do dente é uma parte normal do processo de envelhecimento e não é evitável (Silva et al., 2016).

A situação de saúde bucal encontrada nos idosos atualmente é reflexo de uma prática de saúde mutiladoras e não preventiva. Desse modo, as perdas dentárias constituem uma marca da desigualdade social, diminuindo a capacidade mastigatória, dificultando o consumo de diversos alimentos e repercutindo em sua saúde sistêmica (Silva et al., 2016). A saúde oral contribui consideravelmente para a qualidade de vida e afeta diretamente questões físicas, psicológicas e sociais, como por 
exemplo, dificuldade de se alimentar, redução da mastigação, estética deficiente, dificuldade de socialização, além de acarretar constrangimento e desconfortos emocionais (Burci et al., 2020).

\section{Considerações Finais}

Durante as últimas décadas, ocorreu uma redução na incidência e prevalência de perda dentária / edentulismo em muitos países, especificamente na população de idosos. No entanto, apesar dessa tendência de declínio, o número médio de dentes perdidos acompanha o aumento da idade.

A perda dos elementos dentários em idosos tem sua maior relação com a presença de cárie e o agravamento da doença periodontal, mas também pode ter influência das mais diversas condições sistêmicas apresentada pelos idosos.

A perda dentária compromete o funcionamento do sistema mastigatório, tendo relação direta com o estado nutricional, assim como com o estado cognitivo que por sua vez acarreta em uma menor atenção à higiene oral, ainda mais agravada pela falta de autonomia para execução das atividades rotineiras.

É extremamente necessário o incentivo a políticas públicas voltadas a ações preventivas direcionadas ao público idoso, tanto das doenças sistêmicas como da cavidade oral. Faz-se necessário incentivar ações preventivas e, quando necessário, apoio familiar / sanitário, como por exemplo o incentivo as visitas regulares ao dentista. Nos casos em que já houver um maior comprometimento, é imprescindível um tratamento reabilitador para que melhore as funções relacionadas à cavidade oral (estética, mastigação, nutrição, deglutição, sociabilidade) assim como melhorar o prognóstico de alguns quadros sistêmicos e promover uma melhor qualidade de vida.

No entanto, sugere-se a realização de ensaios clínicos randomizados com amostras significativas para verificação do real papel de cada condição local e da influência das condições sistêmicas sobre a perda dentária em idosos.

\section{Referências}

Burci, L. M., Zanin, S. M. W., Miguel, O.G., Souza, A.W., Dias, J. F. G., \& Miguel, M. D. (2020). Comparação entre dois instrumentos de avaliação de qualidade de vida em pacientes idosos: a influência da perda de dentes e fatores sociodemográficos. Brazilian dental science, 23(2), 1-9.

Catão, M. H. C. V. \& Xavier A. F. C. (2011). O impacto das alterações do sistema estomatognático na nutrição do idoso. Revista de Atenção à Saúde - RAS, 9(29), 73-78. https://doi.org/10.13037/rbcs.vol9n29.1349.

Chagas, A. M. \& Rocha, E. D. (2012). Aspectos fisiológicos do envelhecimento e contribuição da Odontologia na saúde do idoso. Revista Brasileira de Odontologia, 69(1), 94-96.

Dahl, K. E., Calogiuri G., \& Jönsson B. (2018). Perceived oral health and its association with symptoms of psychological distress, oral status and sociodemographic characteristics among elderly in Norway. BMC Oral Health, 18(1), 93. doi: 10.1186/s12903-018-0556-9.

Dioguardi, M., Gioia, G. D., Caloro, G. A., Capocasale, G., Zhurakivska, K., Troiano, G., Russo, L. L., \& Muzio L. L. (2019). The Association between Tooth Loss and Alzheimer's Disease: a Systematic Review with Meta-Analysis of Case Control Studies. Dentistry Journal, 7(2), 49. doi: 10.3390/dj7020049.

Ferrira O. G. L., Maciel S. C., Silva A. O., Sá, R. C. N., \& Moreira, M. A. S. P. (2010). Significados atribuídos ao envelhecimento: idoso, velho e idoso ativo. Psico-USF, 15(3), 357-364.

Figueiredo, M. S., Carvalho, F. S., \& Carvalho, C. A. P. (2019). Impacto da perda dentária na qualidade de vida de idosos com transtornos psiquiátricos. Arquivos em Odontologia,55(e19). Recuperado de https://periodicos.ufmg.br/index.php/arquivosemodontologia/article/view/12490

Firmo, J. O. A., Peixoto, S. V., Souza, G. A., \& Loyola Filho, A. I. (2020). Evolução das publicações em saúde do idoso na revista Ciência \& Saúde Coletiva. Revista Ciência \& Saúde Coletiva, 25(12). https://doi.org/10.1590/1413-812320202512.16662020

Freitas Junior, A. C., Almeida, E. O., Antenucci, R. M. F., Gallo, A. K. G., \& Silva, E. M. M. (2008). Envelhecimento do aparelho estomatognático: alterações fisiológicas e anatômicas. Revista de Odontologia de Araçatuba, 29(1), 47-52.

Kim, Y-T., Choi, J. K., Kim, D-H., Jeong, S-N., \& Lee, J-H. (2019). Association between health status and tooth loss in Korean adults: longitudinal results from the National Health Insurance Service-Health Examinee Cohort, 2002-2015. Journal of Periodontal \& Implant Science, 49(3), 158-170. doi: $10.5051 /$ jpis.2019.49.3.158

Konishi, M., Verdonschot, R. G., \& Kakimoto, N. (2020). An investigation of tooth loss factors in elderly patients using panoramic radiographs. Oral Radiology, 37(3), 436-442. doi: 10.1007/s11282-020-00475-6. 
Lopes, E. N. R., Silva, G. R., Resende, C. C. D., Dietrich, E., Costa, M. D. M. A., \& Dietrich, L. (2021) Prejuízos fisiológicos causados pela perda dentária e relação dos aspectos nutricionais na Odontogeriatria. Research, Society and Development, 10(1), e45810111730.

Melo, L. A., Ferreira, L. M. B. M., Santos, M. M., \& Lima, K. C. (2017). Fatores socioeconômicos, demográficos e regionais associados ao envelhecimento populacional. Revista Brasileira de Geriatria e Gerontologia, 20(4), 493-501.

Macena, W. G., Hermano, L. O., \& Costa, T. C. (2018). Alterações fisiológicas decorrentes do envelhecimento. Revista Mosaicum, 15(27), 223-238. Disponível em: https://revistamosaicum.org/index.php/mosaicum/article/view/64. Acesso em: 16 abr. 2021.

Merchant, A. T. (2017). Low Bone Mineral Density Based on Metacarpal Cortical Bone Area May Predict Tooth Loss in Older Men. The journal of evidencebased dental practice, 17(3), 298-299. doi: 10.1016/j.jebdp.2017.07.003.

Oliveira, A. S. (2019). Transição demográfica, transição epidemiológica e envelhecimento populacional no Brasil. Hygeia - Revista Brasileira de Geografia Médica e da Saúde, 15(32), 69-79.

Pan, M-Y., Hsieh, T-C., Chen, P-H., \& Chen, M. Y. (2019). Factors Associated with Tooth Loss in Postmenopausal Women: A Community-Based CrossSectional Study. International Journal Environmental Research and Public Health, 16(20), 3945.

Penoni, D. C., Fidalgo, T. K., Torres, S. R., Varela, V. M., Masterson, D., \& Leao, A. T. T. (2017). Bone density and clinical periodontal attachment in postmenopausal women: a systematic review and meta-analysis. Journal of Dental Research, 96(3), 261-269.

Pereira, K. C. R., Lacerda, J. T., \& Natal, S. (2017). Avaliação da gestão municipal para as ações de atenção à saúde do idoso. Cadernos de Saúde Pública, 33(4), p.e00208815. https://doi.org/10.1590/0102-311X00208815.

Poisson, P., Laffond, T., Campos, S., Dupuis, V., \& Bourdel-Marchasson, I. (2016). Relationships between oral health, dysphagia and undernutrition in hospitalised elderly patients. Gerodontology, 33(2):161-8. doi: 10.1111/ger.12123.

Ranjan, R., Rout, M., Mishra, M. E., \& Kore, S. A. (2019). Tooth loss and dementia: An oro-neural connection. A cross-sectional study. Journal of Indian Society of Periodontology, 23(2), 158-162. doi: 10.4103/jisp.jisp_430_18.

Savić Pavičin, I., Dumančić, J., Jukić, T., \& Badel, T. (2017). The relationship between periodontal disease, tooth loss and decreased skeletal bone mineral density in ageing women. Gerodontology, 34(4), 441-445. doi: 10.1111/ger.12290.

Shen, X., Chen, X., Huang, J., Xu, R., Cheng, J., \& Jiang, H. (2020). Age-dependent role of SIRT6 in jawbone via regulating senescence and autophagy of bone marrow stromal cells. Journal of Molecular Histology, 5(1), 67-76. doi: 10.1007/s10735-020-09857-w.

Silva, Luciana Terezinha. (2011). Alterações bucais do envelhecimento e implicações para a atenção odontológica. TCC apresentado ao Curso de Atenção Básica em Saúde da Família da Universidade Federal de Minas Gerais. 42 fls,

Silva, C. R. F., Pedrosa, M. S., Ferro, F. E. D., \& Ferro, L. B. (2016). Condição de saúde bucal de idosos residentes em instituições de lona permanência em Teresinha - PI. Salusvita, Bauru, 35(2), 219-232.

Tanaka, R.., Tanaka, T., Yeung, A. W. K., Taguchi, A., Katsumata, A., \& Bornstein, M. M. (2020). Mandibular Radiomorphometric Indices and Tooth Loss as Predictors for the Risk of Osteoporosis using Panoramic Radiographs. Oral Health \& Preventive dentistry, 18(1), 773-782. doi: 10.3290/j.ohpd.a45081.

Taguchi, A., Shiraki, M., Tanaka, S., Ohshige, H., \& Nakamura, T. (2019). Improved periodontal disease and prevention of tooth loss in osteoporosis patients receiving once-yearly zoledronic acid: a randomized clinical trial. Menopause, 26(11), 1277-1283. doi: 10.1097/GME.0000000000001393.

Takeuchi, K., Ohara, T., Furuta, M., Takeshita, T., Shibata, Y., Hata, J., Yoshida, D., Yamashita, Y., \& Ninomiya, T. (2017). Tooth Loss and Risk of Dementia in the Community: the Hisayama Study. Journal of the American Geriatrics Society, 65(5), e95-e100. doi: 10.1111/jgs.14791.

Whittemore R. \& Knafl K. (2005). The integrative review: update methodology. Journal of Advanced Nursing, 52(5):546-53. doi:10.1111/j.13652648.2005.03621.x. 